\title{
Removing Independently Even Crossings
}

\author{
Michael J. Pelsmajer ${ }^{1, \star}$, Marcus Schaefer ${ }^{2}$, and Daniel Štefankovič ${ }^{3}$ \\ 1 DePaul University, Chicago, IL 60604, USA \\ mschaefer@cs . depaul .edu \\ 2 Illinois Institute of Technology, Chicago, IL 60616, USA \\ pelsmajer@iit.edu \\ 3 University of Rochester, Rochester, NY 14627, USA \\ stefanko@cs.rochester.edu
}

\begin{abstract}
We show that $\operatorname{cr}(G) \leq\left(\begin{array}{c}2 \operatorname{iocr}(G) \\ 2\end{array}\right)$ settling an open problem of Pach and Tóth 5[1. Moreover, $\operatorname{iocr}(G)=\operatorname{cr}(G)$ if $\operatorname{iocr}(G) \leq 2$.
\end{abstract}

\section{Crossing Numbers}

Pach and Tóth point out in "Which Crossing Number is It Anyway?" that there have been many different ideas on how to define a notion of crossing number including - using current terminology - the following (see [613]):

crossing number: $\operatorname{cr}(G)$, the smallest number of crossings in a drawing of $G$, pair crossing number 1$] \operatorname{pcr}(G)$, the smallest number of pairs of edges crossing in a drawing of $G$,

odd crossing number: $\operatorname{ocr}(G)$, the smallest number of pairs of edges crossing oddly in a drawing of $G$.

We make the typical assumptions on drawings of a graph: there are only finitely many crossings, no more than two edges cross in a point, edges do not pass through vertices, and edges do not touch. (For a detailed discussion see [13.) What about adjacent edges though? Do we allow them to cross or not? Do we count their crossings? Tutte [17] wrote "We are taking the view that crossings of adjacent edges are trivial, and easily got rid of." While this is true for the standard crossing number, it is not at all obvious for other variants. Székely [13] later commented "We interpret this sentence as a philosophical view and not a mathematical claim."

In [5], Pach and Tóth suggest a systematic study of this issue (see also [1, Section 9.4]): they introduce two rules that can be applied to any notion of crossing number. "Rule +" restricts the drawings to drawings in which adjacent edges are not allowed to cross. "Rule -" allows crossings of adjacent edges, but does not count them towards the crossing number. Pairing ocr, pcr, and cr with any of these two rules gives a total of eight possible variants (

\footnotetext{
* Partially supported by NSA Grant H98230-08-1-0043.

${ }^{1}$ Recently, the book by Tao and $\mathrm{Vu}[15$, on additive combinatorics defined the crossing number as pcr.
} 
mentioned above); one of them has its own name: iocr $:=$ ocr $_{-}$, the independent odd crossing number, introduced by Tutte. The figure below is based on a similar figure from [1].

\begin{tabular}{l||l|l|l} 
Rule + & ocr $_{+}$ & pcr $_{+}$ & \multirow{2}{*}{$\mathrm{cr}$} \\
\hline & ocr & pcr & \\
\hline Rule - & iocr $=$ ocr $_{-}$ & pcr $_{-}$ & $\mathrm{cr}_{-}$
\end{tabular}

Not much is known about the relationships between these crossing number variants, apart from what immediately follows from the definitions: the values in the display increase monotonically as one moves from the left to the right and from the bottom to the top. Even $\mathrm{cr}=\mathrm{cr}_{-}$is open. Pach and Tóth did show that $\operatorname{cr}(G) \leq\left(\begin{array}{c}2 \operatorname{ocr}(G) \\ 2\end{array}\right)$, and this implies that five of the variants, namely $\mathrm{ocr}_{+}$, ocr, $\mathrm{pcr}_{+}, \mathrm{pcr}$, and cr cannot be arbitrarily far apart, but the result does not cover the "Rule -" variants. There are examples of graphs for which ocr and pcr differ 1216. Valtr [18] showed that $\operatorname{cr}(G)=O(\operatorname{pcr}(G) / \log \operatorname{pcr}(G))$, which was improved by Tóth [16] to $\operatorname{cr}(G)=O\left(\operatorname{pcr}(G) / \log ^{2} \operatorname{pcr}(G)\right)$.

In this paper, we show that all eight variants are within a square of each other:

Theorem 1. $\operatorname{cr}(G) \leq\left(\begin{array}{c}2 \operatorname{iocr}(G) \\ 2\end{array}\right)$.

This answers an open problem from [5, Problem 13]; also see [1, Problem 9.4.7]. Pach and Tóth asked whether there are functions $f, g, h$, so that $\operatorname{cr}(G) \leq f$ $\left(\operatorname{cr}_{-}(G)\right), \operatorname{pcr}(G) \leq g\left(\operatorname{pcr}_{-}(G)\right)$, and $\operatorname{ocr}(G) \leq h(\operatorname{iocr}(G))$ for all graphs $G$. Theorem 1 implies that $f=g=h=\left(\begin{array}{c}2 x \\ 2\end{array}\right)$ will do, but this is probably not the optimal choice for $f, g$, and $h$, and quite possibly not for bounding cr in terms of iocr either.

Theorem 1 immediately implies that $\operatorname{iocr}(G)=\operatorname{cr}(G)$ if $\operatorname{iocr}(G) \leq 1$. We can improve this result:

Theorem 2. If $\operatorname{iocr}(G) \leq 2$, then $\operatorname{cr}(G)=\operatorname{iocr}(G)$.

The proof of Theorem 2 is too long to be included in this short note and will be contained in the journal version of the paper. It is based on an analysis of the "odd configurations" that can occur in a drawing. We performed such an analysis for ocr so that we could show that $\operatorname{ocr}(G)=\operatorname{cr}(G)$ if $\operatorname{ocr}(G) \leq 3[8$. The proof of Theorem 2 is much harder, since a bound on $\operatorname{iocr}(G)$ does not imply any a priori bound on the number of edges crossing some other edge oddly. Indeed, the following problem is open:

Is there a function $f$ so that every graph $G$ has a drawing with independent odd crossing number $\operatorname{iocr}(G)$ and at most $f(\operatorname{iocr}(G))$ crossings?

For $\operatorname{ocr}(G)$ such a result can be established; the best upper bound $f$ known in this case is exponential [1].

Theorem 2 generalizes the Hanani-Tutte theorem, which states that $\operatorname{iocr}(G)=$ 0 implies that $\operatorname{cr}(G)=0$ [217]. There are aspects of the Hanani-Tutte theorem 
which are still not well understood; for example, to what extent it relies on the underlying surface. Only recently was it extended to the projective plane [7, that is, it was shown that $\operatorname{iocr}_{N_{1}}(G)=\operatorname{cr}_{N_{1}}(G)$ if $\operatorname{iocr}_{N_{1}}(G)=0$. However, it is not clear how to extend this to the case that $\operatorname{iocr}_{N_{1}}(G) \leq 1$ or how to prove the Hanani-Tutte theorem for surfaces beyond the projective plane. We do know that $\operatorname{ocr}_{S}(G)=\operatorname{cr}_{S}(G)$ if $\operatorname{ocr}_{S}(G) \leq 2$ for arbitrary surfaces $S$ [10].

The independent odd crossing number is implicit in Tutte's paper "Toward a Theory of Crossing Number" [17] which attempts to build an algebraic foundation for the study of the standard crossing number. From an algebraic point of view, ocr and iocr are more convenient parameters than the standard crossing number; for example, the paper by Pach and Tóth [6] shows that iocr $\leq k$ can be recast as a vector-space problem. Tutte's algebraic approach has been continued by Székely [13 14 and, along different lines, Norine [3] and van der Holst [19]. Theorem 1 justifies the approach of studying standard crossing number via independent odd crossing number, by showing that they are not too far apart; indeed, it is tempting to conjecture that $\operatorname{cr}(G)=O(\operatorname{iocr}(G))$. And in spite of the fact that determining the independent odd crossing number of a graph is NP-complete 9], we feel that due to its algebraic nature it offers an intriguing and underutilized alternative approach to algorithmic aspects of crossing number problems.

\section{Removing Even More Crossings}

An edge in a drawing of a graph is odd if it is part of an odd pair, which is a pair of edges that cross an odd number of times. Edges that are not odd are even, and they cross every edge an even number of times (possibly zero times). An edge in a drawing is independently odd if it is part of an independently odd pair, which is a pair of non-adjacent edges that cross an odd number of times. Edges that are not independently odd are independently even, or iocr-0 for short; an iocr-0 edge crosses all non-adjacent edges evenly (possibly zero times), while it may cross adjacent edges arbitrarily. Throughout this paper graphs are simple, that is, they have no loops or multiple edges, unless we say otherwise.

Pach and Tóth showed that if $E$ is the set of even edges in a drawing $D$ of $G$, then $G$ can be redrawn so that all edges in $E$ are crossing-free. As a corollary, they obtained $\operatorname{cr}(G) \leq\left(\begin{array}{c}2 \operatorname{ocr}(G) \\ 2\end{array}\right)$ 6]. We strengthen the Pach-Tóth result to the case that $E$ is the set of independently even edges. According to Pach and Sharir 4, this has been conjectured.

Lemma 1. If $D$ is a drawing of a graph $G$ in the plane, then $G$ has a redrawing in which the independently even edges of $D$ are crossing-free, and every pair of edges crosses at most once.

Proof (Theorem 11). Start with a drawing $D$ of $G$ that realizes $\operatorname{iocr}(G)$, that is, $\operatorname{iocr}(D)=\operatorname{iocr}(G)$. If $F$ is the set of independently odd edges in $D$, then $|F| \leq$ 2 iocr $(D)$. By Lemma 1, there is a drawing of $G$ with at most $\left(\begin{array}{c}|F| \\ 2\end{array}\right)$ crossings. 
To prove Lemma 1, we adapt the following result (a different strengthening of the Pach-Tóth result) from even/odd edges to iocr-0/independently odd edges. The rotation of a vertex is the cyclic order in which edges leave the vertex in a drawing, read clockwise. The rotation system of a drawing is the collection of all vertex rotations.

Lemma 2 (Pelsmajer, Schaefer, and Štefankovič [8]). If $D$ is a drawing of $G$ in the plane and $F$ is the set of odd edges in D, then $G$ has a redrawing with the same rotation system, in which $G-F$ is crossing-free and there are no new pairs of edges that cross an odd number of times.

Splitting a vertex means creating two copies of the vertex with an edge between them so that any edge incident to the original vertex is incident to exactly one of the two copies. (According to this definition, it makes sense to talk about the edges of the original graph occurring in the graph after a vertex split, even though the incidences of edges will change.) With this operation we can now state our analogue of Lemma 2 for iocr-0/independently odd edges.

Lemma 3. If $D$ is a drawing of $G$ in the plane, and $F$ is the set of independently odd edges in $D$, then one can apply a sequence of vertex splits to obtain a graph $G^{\prime}$ with drawing $D^{\prime}$ and the set $F^{\prime}$ of independently odd edges in $D^{\prime}$, such that (1) there are no new independent odd pairs (and hence $F^{\prime} \subseteq F$ ), and (2) every edge of $G^{\prime}-F^{\prime}$ that is not a cut-edge of $G^{\prime}-F^{\prime}$ is crossing-free in $D^{\prime}$.

An edge is a cut-edge if and only if it belongs to no cycles, so Property (2) can be restated as saying that the union of cycles in $G^{\prime}-F^{\prime}$ is crossing-free in $D^{\prime}$. Also, if $Y$ is the set of cut-edges of $G^{\prime}-F^{\prime}$, then $G^{\prime}-\left(F^{\prime} \cup Y\right)$ is crossing-free in $D^{\prime}$.

Proof (Lemma 3). Fix a drawing $D$ of $G=(V, E)$ and let $F$ be the set of independently odd edges in $D$. We establish the theorem by induction. We need to modify $G$ during the proof by splitting vertices, hence we will use induction over the weight

$$
w(G):=\sum_{v \in V} d(v)^{3}
$$

of $G$ where $d(v)$ the degree of $v$ in $G$. For two graphs of the same weight, we induct over the number of cycles that are not crossing-free.

Suppose that $C$ is a crossing-free cycle, with a vertex $u$ that is incident to more than one edge on the same side of $C$. We modify the graph by splitting $u$ into $u_{1}$ (replacing $u$ on $C$ ) and $u_{2}$ (attached to the edges on the side with more than one edge) and inserting an edge between $u_{1}$ and $u_{2}$. This operation results in a graph $G^{\prime}$ with smaller weight and it does not create new independently odd edges (since edges in the exterior of $C$ cannot cross edges on the interior, as all edges along $C$ are crossing-free). We can now apply induction to $G^{\prime}$ to obtain the result. Thus, we may assume that for every vertex $u$ in a crossing-free cycle $C, u$ is incident to at most one edge on the interior of $C$ and at most one edge 
on the exterior of $C$. It follows that any two edges incident to a vertex $u$ in a crossing-free cycle do not cross.

Suppose that $C$ is a cycle made up of iocr-0 edges only, and $C$ is not crossingfree. At each vertex $u$ of $C$ we can ensure that the two edges of $C$ incident at $u$ (say, $e$ and $f$ ) cross evenly by modifying the rotation at $u$ and redrawing $G$ close to $u$. The rotation of the remaining edges at $u$ can then be changed so that each of them crosses $e$ and $f$ evenly. After the redrawing, all the edges of $C$ are even and we can apply Lemma 2 to remove all crossings with edges of $C$ without changing the rotation system or adding new pairs of edges that cross oddly. Now $C$ is crossing-free, and no new independently odd pairs have been added. Suppose that $C^{\prime}$ is a cycle that was crossing-free before the redrawing. If $C$ and $C^{\prime}$ share a vertex $u$, then the rotation at $u$ is not modified when making $C$ crossing-free, so the drawing of $C^{\prime}$ near $u$ is unchanged. $C^{\prime}$ remains crossing-free under the redrawing of Lemma 2 too. Thus we have decreased the number of cycles that are not crossing-free.

We can therefore assume that any cycle consisting of iocr-0 edges is crossingfree. Any other iocr-0 edge is a cut-edge in the graph restricted to iocr-0 edges.

\section{With Lemma 3, we can now prove Lemma 1}

Proof (Lemma 1). Fix a drawing $D$ of $G$ and let $F$ be the set of independently odd edges in $D$. Apply Lemma 3 to obtain a graph $G^{\prime}$ with drawing $D^{\prime}$, let $F^{\prime}$ be the set of independently odd edges in $D^{\prime}$, and let $Y$ be the set of cut-edges in $G^{\prime}-F^{\prime}$. Since $F^{\prime} \cup Y$ contains all crossings in $D^{\prime}, G^{\prime}-\left(F^{\prime} \cup Y\right)$ is crossing-free in $D^{\prime}$ and we can let $S$ be the set of its faces. Within each face of $S$, the edges of $Y$ contained in it can be redrawn one-by-one without creating any crossings, since no edge of $Y$ can complete a path that cuts a face in two (because then it would be part of a cycle in $G^{\prime}-F^{\prime}$, which contradicts it being a cut-edge of $\left.G^{\prime}-F^{\prime}\right)$. This yields a crossing-free drawing of $G^{\prime}-F^{\prime}$, and each of its faces corresponds to a face of $S$, with boundary formed from the boundary of the face of $S$ and the edges of $Y$ in that face. Therefore, each edge of $F^{\prime}$ still has both endpoints incident to a face. Within each face, all such edges of $F^{\prime}$ can be drawn so that every pair of edges crosses at most once.

Since $G^{\prime}$ was obtained from $G$ by a sequence of vertex splits, $G$ can be obtained from $G^{\prime}$ by a sequence of edge contractions. The edges in $E\left(G^{\prime}\right)-E(G)$ are crossing-free, so applying that sequence of contractions to the current drawing of $G^{\prime}$ yields a drawing of $G$ in which $G-F^{\prime}$ is crossing-free and each pair of edges in $F^{\prime}$ crosses at most once. Since $F^{\prime} \subseteq F$, this completes the proof.

\section{References}

1. Brass, P., Moser, W., Pach, J.: Research Problems in Discrete Geometry. Springer, New York (2005)

2. Chojnacki (Haim Hanani), C.: Über wesentlich unplättbare Kurven im dreidimensionalen Raume. Fundamenta Mathematicae 23, 135-142 (1934) 
3. Norine, S.: Pfaffian graphs, T-joins and crossing numbers. Combinatorica 28(1), 89-98 (2008)

4. Pach, J., Sharir, M.: Combinatorial geometry and its algorithmic applications: The Alcalá lectures. Mathematical Surveys and Monographs, vol. 152. American Mathematical Society, Providence (2009)

5. Pach, J., Tóth, G.: Thirteen problems on crossing numbers. Geombinatorics 9(4), 194-207 (2000)

6. Pach, J., Tóth, G.: Which crossing number is it anyway? J. Combin. Theory Ser. B 80(2), 225-246 (2000)

7. Pelsmajer, M.J., Schaefer, M., Stasi, D.: Strong Hanani-Tutte on the projective plane. SIAM Journal on Discrete Mathematics 23(3), 1317-1323 (2009)

8. Pelsmajer, M.J., Schaefer, M., Štefankovič, D.: Removing even crossings. J. Combin. Theory Ser. B 97(4), 489-500 (2007)

9. Pelsmajer, M.J., Schaefer, M., Štefankovič, D.: Crossing number of graphs with rotation systems. In: Hong, S.-H., Nishizeki, T., Quan, W. (eds.) GD 2007. LNCS, vol. 4875, pp. 3-12. Springer, Heidelberg (2008)

10. Pelsmajer, M.J., Schaefer, M., Štefankovič, D.: Removing even crossings on surfaces. European Journal of Combinatorics 30(7), 1704-1717 (2009)

11. Pelsmajer, M.J., Schaefer, M., Štefankovič, D.: Crossing numbers and parameterized complexity. In: Hong, S.-H., Nishizeki, T., Quan, W. (eds.) GD 2007. LNCS, vol. 4875, pp. 31-36. Springer, Heidelberg (2008)

12. Pelsmajer, M.J., Schaefer, M., Štefankovič, D.: Odd crossing number and crossing number are not the same. Discrete Comput. Geom. 39(1), 442-454 (2008)

13. Székely, L.A.: A successful concept for measuring non-planarity of graphs: the crossing number. Discrete Math. 276(1-3), 331-352 (2004)

14. Székely, L.A.: An optimality criterion for the crossing number. Ars Math. Contemp. 1(1), 32-37 (2008)

15. Tao, T., Vu, V.: Additive combinatorics. Cambridge Studies in Advanced Mathematics, vol. 105. Cambridge University Press, Cambridge (2006)

16. Tóth, G.: Note on the pair-crossing number and the odd-crossing number. Discrete Comput. Geom. 39(4), 791-799 (2008)

17. Tutte, W.T.: Toward a theory of crossing numbers. J. Combinatorial Theory 8 , 45-53 (1970)

18. Valtr, P.: On the pair-crossing number. In: Combinatorial and Computational Geometry. Math. Sci. Res. Inst. Publ., vol. 52, pp. 569-575. Cambridge University Press, Cambridge (2005)

19. van der Holst, H.: Algebraic characterizations of outerplanar and planar graphs. European J. Combin. 28(8), 2156-2166 (2007) 